\title{
A STUDY FOR AEROBIC MICROBIOLOGICAL PROFILE OF BILE IN PATIENTS UNDERGOING GALLBLADDER SURGERY AND FOR ITS COMPLICATIONS
}

\author{
Praful Bhagat ${ }^{1}$, Atul Mahajan², Satish Kumar ${ }^{3}$, Anupam Nanda ${ }^{4}$ \\ ${ }_{1}^{1} 3^{r d}$ Year Junior Resident, Department of Surgery, Dr. Rajendra Prasad Government Medical College, Tanda, Kangra, (HP). \\ 2 Professor, Department of Surgery, Dr. Rajendra Prasad Government Medical College, Tanda, Kangra, (HP). \\ ${ }^{3}$ Assistant Professor, Department of Surgery, Dr. Rajendra Prasad Government Medical College, Tanda, Kangra, (HP). \\ ${ }^{4}$ Senior Resident, Department of Surgery, Dr. Rajendra Prasad Government Medical College, Tanda, Kangra, (HP).
}

\begin{abstract}
\section{BACKGROUND}

Cholelithiasis is the most common disease found in Northern India. Cholecystectomy is the most common operation performed in our institution. Several studies showed a relationship between intra-operative bile culture and post-operative complications, but in our study, we did not find any such relations.

The aim of this study is to study aerobic microbiological profile of bile in patients undergoing gallbladder surgeries and its relationship with post-operative complications.
\end{abstract}

\section{MATERIALS AND METHODS}

The study population included patients undergoing Gallbladder surgery during one-year period from September 2015 to August 2016. A detailed history was taken from the patient or from the attendant of patient and recorded in a predesigned proforma. Intra-operatively, bile was aspirated from the gallbladder and sent for culture and sensitivity before performing cholecystectomy. All patients were followed till 30 days after surgery for any post-operative complications like abdominal pain, fever, surgical site infections, wound dehiscence or any other complication.

\section{RESULTS}

Out of total 339 patients, bile culture was positive in 34 patients (10.03\%), whereas culture was negative in 305 patients (89.97\%). E. coli was the most common organism found in bile cultures in 16 patients (47.05\%). Post-operative complications developed in 25 patients $(07.37 \%)$ out of total 339 patients. Post-operative surgical site infection was observed in 23 patients out of 305 patients $(07.54 \%)$ in whom bile culture was negative and observed in 02 patients out of 34 in whom bile culture was positive (05.88\%).

\section{CONCLUSION}

In our study, the most common indication for cholecystectomy was gallstone disease. In the study, the presence of bacteria in gallbladder bile cultures was not significant to the development of surgical wound infection after cholecystectomy contrary to some of the studies, which have shown the relationship between bactibilia and post-operative complications.

\section{KEYWORDS}

Cholelithiasis, Bactibilia, Cholecystectomy.

HOW TO CITE THIS ARTICLE: Bhagat P, Mahajan A, Kumar S, et al. A study for aerobic microbiological profile of bile in patients undergoing gallbladder surgery and for its complications. J. Evolution Med. Dent. Sci. 2017;6(53):4002-4006, DOI: 10.14260/Jemds/2017/865

\section{BACKGROUND \\ Benign diseases of biliary tract are the most common surgical problems world over. Majority of the gallbladder diseases are caused by inflammation due to irritation of gallbladder wall known as cholecystitis and further it can be acute or chronic. Gallstone disease is the commonest of all gallbladder disease along with its complications like mucocele of gallbladder, empyema of gallbladder, gangrene of gallbladder, etc. Other diseases of the gallbladder includes gallbladder polyps, acalculous cholecystitis and sclerosing cholecystitis and gallbladder cancer.}

Financial or Other, Competing Interest: None.

Submission 29-05-2017, Peer Review 23-06-2017,

Acceptance 28-06-2017, Published 03-07-2017.

Corresponding Author:

Dr. Atul Mahajan,

Anand Bhawan

Dane Road, Dharamshala Dist.,

Kangra-176215, H. P.

E-mail: dratulmahajan@yahoo.co.in

DOI: $10.14260 /$ jemds $/ 2017 / 865$

\section{(c) $(\mathrm{i})(\mathrm{FP}$}

Autopsy reports have shown a prevalence of gallstones from 11 to $36 \%$. Several factors like supersaturation of bile, gallbladder hypomotility, accelerated nucleation and contamination of bile have been implicated in the causation of gallstones. Infective factors seem to be a major cause of formation of gallstones.

Bacteria are commonly found in inflamed gallbladder of patients with cholelithiasis, whereas evidence suggests that normal bile is sterile. Several reasons for biliary tract infection have been implicated, e.g. ascending infection.

Due to reflux of duodenal contents, blood-borne infection and infection spread through the portal-venous channels. Ascending infection from the duodenum is thought to be the primary mechanism, by which bacteria enter the bile and the percentage of bactibilia increases to $3 \%$ in patients with gallstones and to $\approx 30 \%$ in patients with common bile duct stones. ${ }^{1,2}$ The multiplication of biliary micro-organisms combined with obstruction or manipulation (Surgical or NonSurgical) leads to occurrence of various biliary infections such as complicated cholecystitis, cholangitis, sepsis and surgical wound infections. ${ }^{3}$ The biliary infection is caused by 
various types of organisms ranging from aerobic gram positive to gram negative to anaerobic organisms. Aerobic organisms cause $94 \%$ of biliary tract infections, which includes Escherichia coli, Klebsiella species and Enterococcus faecalis which constitute the normal intestinal flora. Anaerobes, particularly Bacteroides and Clostridia may be isolated from approximately $40 \%$ of infected bile samples.

It is well known that bactibilia is a common finding in patients with risk factors such as biliary obstruction, age of more than 70 years, acute cholecystitis, common bile duct stones, cholangitis and non-functioning gallbladders. ${ }^{4}$

Organisms may be cultured from bile obtained from gallbladder and common bile duct or from liver biopsy tissue in normal patients. Although, these organisms may represent the normal hepatobiliary flora, some may be caused by contamination of specimen during collection. Gallbladder bile from patients with acute and chronic cholecystitis yields organisms in $30-50 \%$ of patients.

Positive culture is more frequent in acute rather than chronic disease and in those with an obstructed as opposed to patent cystic duct. The incidence of positive culture from bile duct bile is greatest (75 - 100\%) when obstruction is caused by a calculus or stenosis of a surgical anastomosis. 4,5

A correlation between bactibilia and post-surgical complications remains variable and undistinguished. For instance, a definite correlation between bacteria cultured from the bile during surgery and post-operative complications has been established. ${ }^{6}$ Aim of this study was to determine the prevalence of bacteria in bile samples of the patients undergoing gallbladder surgeries for cholelithiasis, gallbladder polyps, and early stage carcinoma gallbladder and to correlate bactibilia and post-operative complications.

\section{MATERIALS AND METHODS}

The proposed descriptive study was conducted at Dr. RPGMC at Tanda in Dept. of Surgery over a period of one year from September 2015 to August 2016.

\section{Inclusion Criteria}

The study population included all patients undergoing gallbladder surgery in the Dept. of Surgery at Dr. RPGMC, Tanda.

\section{Exclusion Criteria}

Patients who had undergone any interventional procedures like ERCP, etc.

The study population included patients undergoing gallbladder surgery during one-year period from September 2015 to August 2016. A detailed history was taken from the patient or from the attendant of patient and recorded in a predesigned proforma. Clinical examination consisted of thorough examination including general physical examination and abdominal examination. All patients underwent biochemical investigations including LFTs, Amylase and Lipase. Ultrasonography abdomen was done in all cases and CECT abdomen (if required).

All patients included in the study were given a single shot of prophylactic antibiotic (cefuroxime + clavulanic acid) at the time of induction.

\section{Bile Collection and Bile Culture Analysis}

Intraoperatively, bile was aspirated from the gallbladder with $10 \mathrm{~mL}$ syringe in open cholecystectomy and using aspiration needle of 18-G in case of laparoscopic cholecystectomy and sent for culture and sensitivity before performing cholecystectomy.

\section{Followup}

All patients were followed till 30 days after surgery for any post-operative complications like abdominal pain, fever, surgical site infections, wound dehiscence or any other complications. Those patients who had complications in our study reported back for appropriate management.

\section{RESULTS}

A total of 339 patients with various gallbladder diseases, who met the inclusion criteria and consented to participate in the study were included in the project. The maximum no. of patients in our study was found between the age group of 41 to 50 years, that is 93 patients $(27.43 \%)$ and the mean age of the patients was 45.48 years. In our study, the mean age in bile culture positive patients was 49.76 years, whereas mean age in bile culture negative patients was 45.01 years.

Out of 339 patients 288 were female (85\%), while 51 were male patients $(15 \%)$ and the percentages of females with positive bile culture was higher $(08.84 \%)$ compared with males $(01.17 \%)$. There were 31 patients $(9.14 \%)$ who had co-morbid conditions; 6 out of 31 patients (19.4\%) with concomitant diseases had positive bile cultures; however, 28 out of 308 patients $(9.1 \%)$ without any concomitant diseases had positive bile culture. 227 patients (65\%) underwent laparoscopic cholecystectomy and 109 patients (31\%) underwent open cholecystectomy, whereas 13 patients (4\%) need conversion from laparoscopic to open cholecystectomy. Positive bile cultures were present in 19 (55.88\%) in open cholecystectomy patients and 15 (44.11\%) in laparoscopic cholecystectomy patients.

Out of total 339 patients bile culture was positive in 34 patients (10.03\%), whereas culture was negative in 305 patients (89.97\%) (Table 1).

\begin{tabular}{|c|c|c|}
\hline & No. of Cases & Percentages \\
\hline $\begin{array}{c}\text { Bile culture } \\
\text { positive cases }\end{array}$ & 34 & 10.03 \\
\hline $\begin{array}{c}\text { Bile culture } \\
\text { negative cases }\end{array}$ & 305 & 89.97 \\
\hline Total & 339 & 100 \\
\hline
\end{tabular}

E. coli was the most common organism found in bile cultures in 16 patients (47.05\%) (Table 2).

\begin{tabular}{|c|c|c|}
\hline Type of Organisms & $\begin{array}{c}\text { No. of } \\
\text { Patients }\end{array}$ & Percentages \\
\hline E. coli & 16 & 47.05 \\
\hline Enterobacter & 4 & 11.76 \\
\hline Klebsiella & 3 & 8.82 \\
\hline Staphylococcus species & 3 & 8.82 \\
\hline Pseudomonas & 3 & 8.82 \\
\hline Mixed (E. coli + Klebsiella) & 2 & 5.88 \\
\hline $\begin{array}{c}\text { Non-fermenting group of } \\
\text { organisms }\end{array}$ & 2 & 5.88 \\
\hline Acinetobacter & 1 & 2.94 \\
\hline
\end{tabular}


Out of 339 patients, post-operative complications developed in 25 patients $(07.37 \%)$. Out of these, postoperative surgical site infection was observed in 23 out of 305 patients $(07.54 \%)$ in whom bile culture was negative on $4^{\text {th }}, 5^{\text {th }}$ and $6^{\text {th }}$ post-operative days respectively (Table 3 ).

\begin{tabular}{|c|c|}
\hline $\begin{array}{c}\text { Bile Culture Negative } \\
\text { Patients }\end{array}$ & $\begin{array}{c}\text { Post-Operative } \\
\text { Complications }\end{array}$ \\
\hline 305 & 23 \\
\hline Table 3. Post-Operative Complications in Bile Culture \\
Negative Patients \\
\hline
\end{tabular}

Post-operative surgical site infection was observed in 02 patients out of 34 , in whom bile culture was positive $(05.88 \%)$ on $5^{\text {th }}$ post-operative day (Table 4$)$.

\begin{tabular}{|c|c|}
\hline $\begin{array}{c}\text { Bile Culture Positive } \\
\text { Patients }\end{array}$ & $\begin{array}{c}\text { Post-Operative } \\
\text { Complications }\end{array}$ \\
\hline 34 & 02 \\
\hline \multicolumn{2}{|c|}{ Table 4. Post-Operative Complications in Bile Culture } \\
Positive Patients
\end{tabular}

There were 32 patients (94.11\%) whose bile culture was positive, but did not develop any post-operative complications and remaining 282 patients (83.18\%) found their bile culture negative without any post-operative complications.

Out of 339 patients, intra-operative bile spillage occurred in 23 patients $(6.78 \%)$, whereas 316 patients had no bile spillage (93.21\%) (Table 5).

\begin{tabular}{|c|c|}
\hline Intraoperative Bile Spillage & No. of Patients \\
\hline Yes & 23 \\
\hline No & 316 \\
\hline Table 5. Intraoperative Bile Spillage \\
\hline
\end{tabular}

Surgical site infections developed in one patient out of 23 (04.34\%), in whom intra-operative bile spillage occurred and in 24 patients out of $316(07.59 \%)$ in whom there was no intra-operative spillage of bile (Table 6).

\begin{tabular}{|c|c|}
\hline Intraoperative Bile Spillage & Surgical Site Infection \\
\hline Present $=23$ & 1 \\
\hline Absent $=316$ & 24 \\
\hline Table 6. Intraoperative Bile Spillage and Surgical Site \\
Infections
\end{tabular}

Out of 23 patients in whom intra-operative bile spillage occurred bile culture was positive in 09 patients, but none of these patients had SSI and 1 patient in whom SSI developed had negative bile culture report (Table 7).

\begin{tabular}{|c|c|c|}
\hline & $\begin{array}{c}\text { Total No. of } \\
\text { Patients }\end{array}$ & $\begin{array}{c}\text { Surgical Site } \\
\text { Infection }\end{array}$ \\
\hline Bile culture positive cases & 09 & 0 \\
\hline Bile culture negative cases & 14 & 01 \\
\hline Table 7. Presence of Intraoperative Bile Spillage with \\
Surgical Site Infection \\
\hline
\end{tabular}

Out of 316 patients in whom there was no intra-operative bile spillage, intraop bile culture report was positive in 25 patients and out of these 25 patients SSI developed in only 2 patients (08\%), whereas remaining 291 patients in which bile culture was negative SSI developed in 22 patients $(07.56 \%)$. (Table 8).

\begin{tabular}{|r|c|c|}
\hline & $\begin{array}{c}\text { Total No. of } \\
\text { Patients }\end{array}$ & $\begin{array}{c}\text { Surgical Site } \\
\text { Infection }\end{array}$ \\
\hline Bile culture positive cases & 25 & 02 \\
\hline Bile culture negative cases & 291 & 22 \\
\hline Table 8. Absence of Intraoperative Bile Spillage with \\
Surgical Site Infection \\
\hline
\end{tabular}

Thereby, meaning that intra-operative bile spillage has got to do nothing with post-operative complications.

The statistical analysis was done using SPSS (Statistical Package for Social Sciences) Version 17.0 and statistical significance was assessed with the help of Pearson ChiSquare test and $\mathrm{p}$-value of $<0.05$ was taken as statistically significant.

\section{DISCUSSION}

In our study the mean age of patients was 45.48 years, which is close to that observed in study done by Hazrah ${ }^{7}$ et al $(43.48$ years). In our study the mean age in bile culture positive patients was 49.76 years, whereas mean age in bile culture negative patients in our study was 45.01 years. There was a significant difference $(\mathrm{p}=0.043)$ when comparing the mean age between groups. Study results show that age is directly proportional in relation to the risk of bacteria being present in the bile, similar to that reported in other studies. So age is the significant pre-operative risk factor associated with positive bile cultures.

In the present study, total no. of female patients were 288 $(85 \%)$ and male patients were $51(15 \%)$, which is similar to Al Harbi ${ }^{8}$ et al $(72.32 \%)$. The percentages of females with positive bile culture was higher $(08.84 \%)$ compared with males $(01.17 \%)$, whereas Velazquez ${ }^{9}$ et al also reported the higher no. of females (33.75\%) in their study as compared to males (16.25\%). This may be a reflection of the fact that more females were included in the study, but there was no difference in relation to the gender between two groups $(\mathrm{p}=$ 0.573).

There were 31 patients $(9.14 \%)$ in the study with comorbid conditions. Only one patient developed surgical site infection post-operatively; 6 out of 31 patients (19.4\%) with concomitant diseases had positive bile cultures; however, 28 out of 308 patients $(9.1 \%)$ without any concomitant diseases had positive bile culture $(p=0.070)$. So there was no significant difference in relation to co-morbid conditions between the two groups in our study.

In our study the positive bile culture in cases of cholelithiasis was found in $10.03 \%$, which coincides with Van Leeuwen ${ }^{10}(16.4 \%)$.

The bile culture positive results were higher in the patients operated by open cholecystectomy (55.88\%) than that compared to laparoscopic cholecystectomy (44.11\%). This is because of slight probability of post-operative infections due to the creation of tiny wounds as well as tissue damage, which is less common in laparoscopic cholecystectomy than open surgery. 
In the present study, the most common organism isolated from bile samples was E. coli (47.05\%) similar to that has been reported in other studies such as Zafar ${ }^{11}(43.75 \%)$ and $\operatorname{Kedar}^{5}$ (38.88\%). Overall, post-operative surgical site infections in our study occurred in 25 out of 339 patients $(07.37 \%)$ which coincides with Abdullah ${ }^{12}$ et al $(7.66 \%)$. Post-operative surgical site infection was found in 2 out of 34 patients $(05.88 \%)$ who had bactibilia, which is close to Van Leeuwen ${ }^{10}$ et al (9\%) and Kedar $^{5}$ et al (10\%). Post-operative surgical site infection was found in 23 patients $(07.54 \%)$ out of 305 patients whose bile culture was sterile which is similar to study done by Uludag 13 et al. The rate of surgical wound infection in our study showed no statistical significance among culture-positive patients compared with culturenegative patients $(\mathrm{p}=0.726)$. Many reports have also indicated that wound infections are not related to bile culture, rupture of gallbladder or spillage of gallbladder stones or bile.7,14 Therefore, the cause of surgical site infection in these 25 patients in our study could be in part explained by various other reasons such as inadequate skin preparation, aseptic manipulation and incomplete homeostasis, etc.

In the present study, bile spillage occurred in total 23 patients $(06.78 \%)$ which is less than that observed in study done by Sansiya ${ }^{15}$ et al (92\%) and Jasim ${ }^{16}$ et al (97\%).

Surgical site infections developed in 1 patient out of 23 $(04.34 \%)$, in whom intra-operative bile spillage occurred which is similar to that observed by Sansiya ${ }^{15}$ et al (4\%).

In our study, surgical site infection developed in 24 out of $316(07.59 \%)$, in whom there was no intra-operative spillage of bile. So, there was no significant difference between postoperative complications and intra-operative bile spillage $(\mathrm{p}=0.565)$.

Out of 23 patients in whom intra-operative bile spillage occurred bile culture was positive in 09 patients, but none of these patients had surgical site infections and 1 patient in whom surgical site infection developed had negative bile culture report $(\mathrm{p}=0.412)$.

Out of 316 patients in whom there was no intra-operative bile spillage intra-operative bile culture report was positive in 25 patients and out of these 25 patients surgical site infections developed in only 2 patients (08\%), whereas remaining 291 patients in which bile culture was negative surgical site infections developed in 22 patients $(07.56 \%)$ $(\mathrm{p}=0.937)$.

So this study shows that intra-operative bile spillage is not related to post-operative surgical site infections.

\section{CONCLUSION}

In our study, the most common indication for cholecystectomy was gallstone disease. In the study, the presence of bacteria in gallbladder bile cultures was not significant to the development of surgical wound infection after cholecystectomy contrary to some of the studies which have shown the relationship between bactibilia and postoperative complications. Moreover, the rate of surgical site infection was not significant in patients with positive bile cultures as compared to patients with negative bile cultures in our study.
In the study, no relationship was observed between intraoperative bile spillage and post-operative complications. The patients in which wound site infections were present following biliary surgery could have been due to endogenous or exogenous contamination. In our study, aerobic organisms were most commonly isolated from bile samples and most commonly isolated bacteria were gram-negative bacteria, i.e. E. coli, Enterobacter and mixed cultures.

\section{REFERENCES}

[1] Csendes A, Burdiles P, Maluenda F, et al. Simultaneous Bacteriologic assessment of bile from gallbladder and common bile duct in Control subjects and patients with gallstones and common duct stones. Arch Surg 1996; 131(4):389-94.

[2] Csendes A, Mitru N, Maluenda F, et al. Counts of bacteria and pyocites of choledochal bile in controls and in patients with Gallstones or common bile duct stones with or without acute cholangitis. Hepatogastroenterology 1996;43(10):800-06.

[3] Peria C, Gudiol F. Cholecystitis and cholangitis: spectram of bacteria and role of antibiotics. Dig Surg 1996; 13: 317-20.

[4] Morris-Stiff GJ, O’Donohue P, Ogunbiyi S, et al. Microbiological assessment of bile during cholecystectomy: is all bile infected? HPB (Oxford) 2007; 9(3):225-8.

[5] Kedar S, Sanjeev S, Rakesh G. Microbiological profile of bile in cholelithiasis And their implication in causing post-operative wound Infections. J Evolution Med and Dental Sci (JEMDS) 2014; 144:78-82.

[6] Jackaman FR, Hilson GR, Smith L. Bile bacteria in patients with benign bile duct stricture. Br J Surg 1980; 67(5):329-32.

[7] Hazrah P, Oahn KTH, Tewari M, et al. The frequency of live bacteria in gallstones. HPB 2004;6(1):28-32.

[8] Al Harbi M, Osoba AO, Mowallad A, et al. Tract microflora in Saudi patients with cholelithiasis. Trop Med Int Health 2001;6(7):570-4.

[9] Velazquez-Mendoza JD, Alvarez-Mora M, VelázquezMorales CA, et al. Bactibilia and surgical site infection after open cholecystectomy. Cir Cir 2010;78(3): 239-43.

[10] Van Leeuwen P, Keeman JN, Butzelaar RM, et al. Correlation between a positive gallbladder culture and subsequent wound infection after biliary surgery-a retrospective study of 840 patients. Neth J Surg 1985; 37(6):179-82.

[11] Malik ZI, Malik MAN, Salahuddin 0, et al. Micro flora of bile aspirates in symptomatic cholelithiasis. JRMC 2009; 13(1):38-40.

[12] Abdullah A, Medhat M, Hamid L, et al. Infection after laparoscopic cholecystectomy: effect of infected bile and infected gallbladder wall. Eur J Surg 2001; 167(4):268-73.

[13] Uludag M, Yetkin G, Citgez B. The role of prophylactic antibiotics in elective Laparoscopic cholecystectomy. Factors influencing wound infection following Laparoscopic cholecystectomy. J Society of Laparoscopic Surg 2009; 13(3):337-41. 


\section{Jemds.com}

[14] Sabry A, Wael W, Waleed O, et al. Antibiotics prophylaxis in Elective laparoscopic cholecystectomy: a prospective study. EJS 2005; 24: 145-51.

[15] Sansiya K, Dodia H, Patel D, et al. Evaluation of gallbladder content spillage in laparoscopic cholecystectomy. IOSR-JDMS 2015; 14(8):105-22.
Original Research Article

[16] Jasim D, Mazin A, Mushtaq C. Spilled gallstones during laparoscopic cholecystectomy: a prospective study. Basrah Journal of Surgery 2011; 2: 123-7. 